\title{
ARTICLE
}

Received 31 Mar 2016 | Accepted 18 Oct 2016 | Published 22 Nov 2016

DOI: $10.1038 /$ ncomms13590 OPEN

\section{Nanomechanical electro-optical modulator based on atomic heterostructures}

\author{
P.A. Thomas ${ }^{1}$, O.P. Marshall ${ }^{1}$, F.J. Rodriguez ${ }^{1}$, G.H. Auton², V.G. Kravets ${ }^{1}$, D. Kundys ${ }^{1}$, Y. Su ${ }^{1} \&$ A.N. Grigorenko ${ }^{1}$
}

Two-dimensional atomic heterostructures combined with metallic nanostructures allow one to realize strong light-matter interactions. Metallic nanostructures possess plasmonic resonances that can be modulated by graphene gating. In particular, spectrally narrow plasmon resonances potentially allow for very high graphene-enabled modulation depth. However, the modulation depths achieved with this approach have so far been low and the modulation wavelength range limited. Here we demonstrate a device in which a graphene/hexagonal boron nitride heterostructure is suspended over a gold nanostripe array. A gate voltage across these devices alters the location of the two-dimensional crystals, creating strong optical modulation of its reflection spectra at multiple wavelengths: in ultraviolet Fabry-Perot resonances, in visible and near-infrared diffraction-coupled plasmonic resonances and in the mid-infrared range of hexagonal boron nitride's upper Reststrahlen band. Devices can be extremely subwavelength in thickness and exhibit compact and truly broadband modulation of optical signals using heterostructures of two-dimensional materials.

\footnotetext{
${ }^{1}$ School of Physics and Astronomy, University of Manchester, Manchester M13 9PL, UK. ${ }^{2}$ School of Computer Science, University of Manchester, Manchester M13 9PL, UK. Correspondence and requests for materials should be addressed to A.N.G. (email: sasha@manchester.ac.uk).
} 
$\mathrm{P}$ lasmonics and the nano-optics of two-dimensional (2D) materials are two of the most rapidly advancing areas in photonics ${ }^{1-3}$. The advent of high-resolution nanofabrication techniques has allowed for the development of plasmonic nanostructures with a broad range of optical responses such as subwavelength confinement of light ${ }^{4}$ and ultra-narrow diffraction-coupled resonances ${ }^{5-7}$ that could lead to applications in waveguiding ${ }^{8}$ and biosensing ${ }^{9}$, respectively. Meanwhile, the extraordinary properties of $2 \mathrm{D}$ materials have generated great interest in the nano-optics community ${ }^{10}$. Graphene has demonstrated remarkable optical properties that include visual transparency defined by the fine structure constant ${ }^{11}$ and gate-tuneable intrinsic plasmons $3,12,13$. More recently, graphene has been combined with other 2D materials such as hexagonal boron nitride $(\mathrm{hBN})$ to form heterostructures ${ }^{14}$ that have already been used to develop light-emitting diodes ${ }^{15}$.

The combination of plasmonic nanostructures with 2D heterostructures shows particular promise ${ }^{3}$. Placing plasmonic nanostructures in $2 \mathrm{D}$ heterostructure stacks allows for much stronger interactions between light and $2 \mathrm{D}$ materials ${ }^{16}$. This approach has been used to produce significant advances in the field of photovoltaics ${ }^{17}$, Raman spectroscopy ${ }^{18}$ and sensing ${ }^{9}$. Arguably the most exciting developments could come in optoelectronics ${ }^{2,3,8}$ - the optical properties of graphene can easily be tuned by applying a gate voltage ${ }^{19}$. Combining graphene with metallic nanostructures capable of supporting ultra-narrow plasmon resonances could potentially lead to the development of compact optical modulators ${ }^{3}$. However, attempts to experimentally realize such a device have met with limited success ${ }^{20-23}$-modulation depths are typically small (or slow for graphene supercapacitors) and influence over higher-energy spectral features requires exceptionally high doping of graphene.

Here we use electromechanical modulation to achieve high modulation depths in a larger frequency range. Indeed, relatively little attention has been paid to the potential for nanoelectromechanical optical effects in either plasmonic or $2 \mathrm{D}$ heterostructure systems ${ }^{24,25}$. We describe a graphene/hBN/gold nanostripe heterostructure with an air gap between the hBN and plasmonic nanostripes. Application of a gate voltage between graphene and the gold nanostripe sublayer leads to a reduction in the air gap height. This in turn changes the effective electric field acting on graphene and hBN, modifying spectral reflection features from mid-ultraviolet to mid-infrared wavelengths. The demonstration of strong modulation (of up to $30 \%$ ) in a $2 \mathrm{D}$ material-based device, particularly over such a broad range of spectral features, is unprecedented. It is important to stress that the volume in which the modulation is achieved is extremely small. For example, in the mid-infrared region (at a wavelength $\lambda$ $\sim 7 \mu \mathrm{m}$ ) the optical interrogation volume is $\sim \lambda^{3} / 10$, the smallest reported so far-approximately three orders of magnitude smaller than reported in ref. 25. Our results open up new possibilities for strong light modulation in optoelectronic devices.

\section{Results}

Sample design. The design of our graphene/hBN/nanoarray modulator is shown in Fig. 1a. The gold plasmonic nanostripe array is separated from the graphene/hBN by an air gap $(d)$. Using the graphene as a broadband, transparent and extremely tough electrical contact, a gate voltage can be applied across the structure. In this work the nanostripe array had a periodicity $a=1,570 \mathrm{~nm}$, stripe width $w=550 \mathrm{~nm}$, stripe height $h_{2}=80 \mathrm{~nm}$ and gold sublayer of thickness $h_{1}=65 \mathrm{~nm}$ (Fig. 1b)-see Methods for device fabrication details. A representative scanning electron microscopy micrograph is shown in Fig. 1c. Such plasmonic nanoarrays can be tuned to give narrow, diffraction-coupled resonances that arise when the wavelengths of diffracted
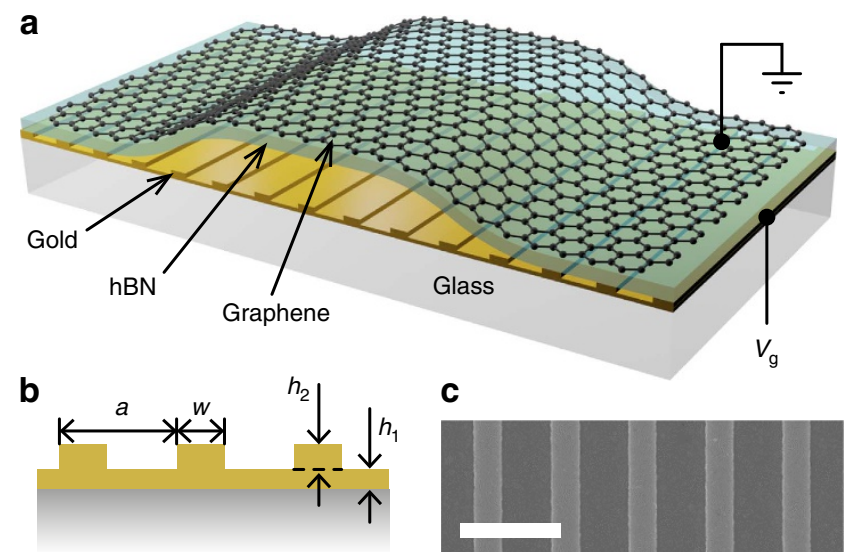

C

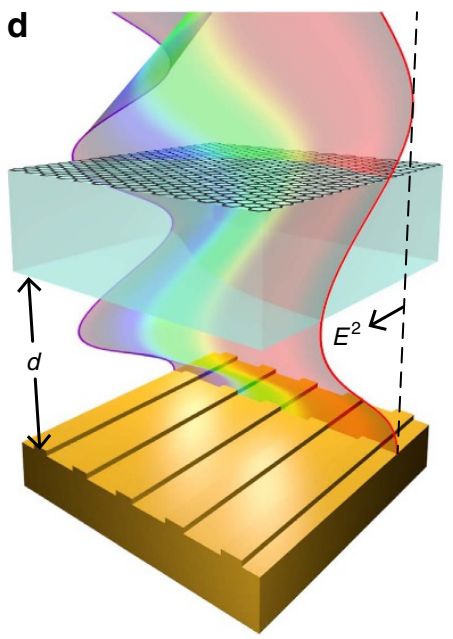

Figure 1 | Nanomechanical electro-optical modulator structure.

(a) Schematic of our device with air gap height $d$. (b) Geometric design parameters for our gold nanostripe array. (c) Representative scanning electron microscopy (SEM) micrograph of the nanostripe array (scale bar $2 \mu \mathrm{m}$ ). (d) The working principle of the device. The coloured wave represents an unperturbed standing wave for different wavelengths observed under reflection from the nanostructured mirror.

light modes, running along the air-substrate boundary (known as Rayleigh cutoff wavelengths), are recaptured as electron oscillations in the plasmonic nanostructures ${ }^{5}$. These resonances can be further narrowed by adding a metallic sublaye ${ }^{26}$. Our nanostructure was designed to produce a narrow plasmon resonance around the telecom wavelength of $\lambda \sim 1.5 \mu \mathrm{m}$, although higher-order diffraction-coupled modes exist throughout the near-infrared and visible spectrum. An hBN flake $(\sim 110 \mathrm{~nm}$ thick) and single-layered graphene were then mechanically exfoliated and transferred on to the plasmonic nanostructure (see Methods). Hexagonal boron nitride is an ideal dielectric for graphene devices because it is an atomically flat crystal with a similar lattice constant to graphene ${ }^{27}$. Within the modulator region of our device the initial air gap between the $\mathrm{hBN}$ and nanostripe array was $\sim 300 \mathrm{~nm}$. An optical image of the completed device is shown in Supplementary Fig. 1. Atomic force microscopy data confirming the device dimensions is shown in Supplementary Fig. 2.

Spectroscopic ellipsometry and reflectometry. Devices were studied using spectroscopic ellipsometry and reflectometry (see Methods). Figure 2a shows the ellipsometric reflection spectrum $(\Psi)$ of a device from the mid-ultraviolet through to the nearinfrared, when illuminated at an incident angle of $\theta=70^{\circ}$. We 
a
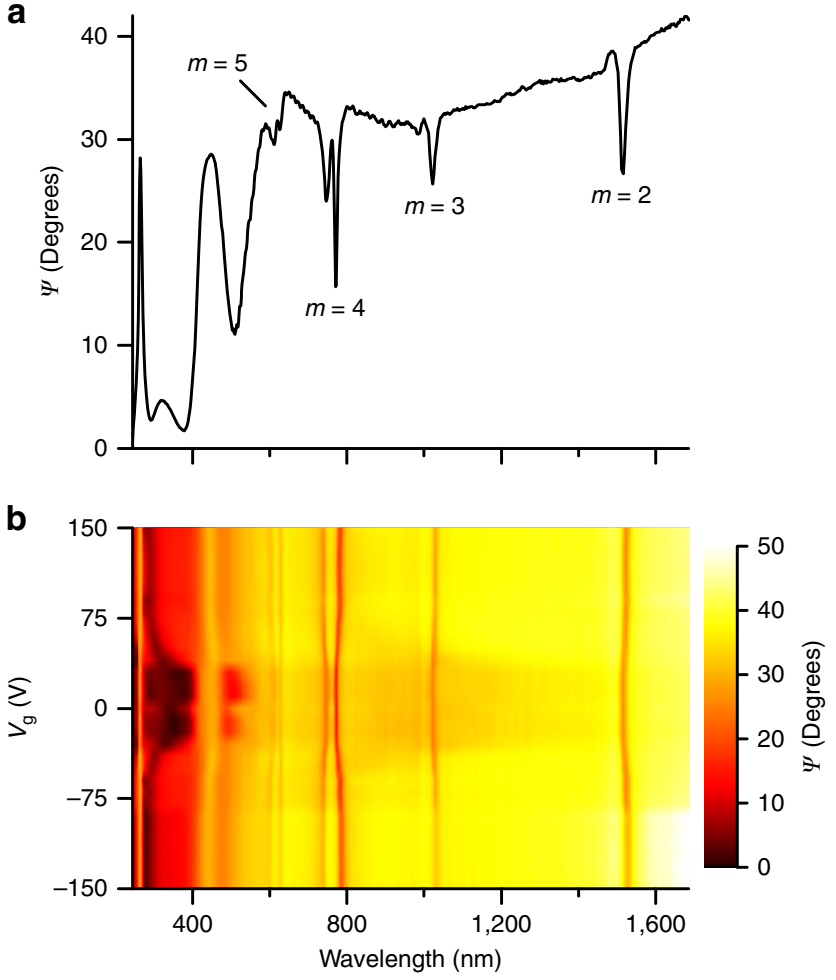

Figure 2 | Device characterization using spectroscopic ellipsometry.

(a) Ellipsometric reflection spectrum $\Psi$ of our graphene/hBN/plasmonic heterostructure $\left(V_{\mathrm{g}}=0 \mathrm{~V}, \theta=70^{\circ}\right)$. (b) Colour map of $\Psi$ as a function of wavelength and $V_{g}$.

attribute the broad absorption peaks in the wavelength range $280 \mathrm{~nm}<\lambda<590 \mathrm{~nm}$ to Fabry-Perot (FP) interference in the air gap, whereas the sharp feature at $\lambda=275 \mathrm{~nm}$ is caused by the complex, multi-peaked ultraviolet absorption spectrum of $\mathrm{hBN}^{28}$. The remaining strong features from $590 \mathrm{~nm}<\lambda<1,600 \mathrm{~nm}$ primarily arise from the nanoarray and correspond to the Rayleigh cutoff wavelengths for air, determined by $\lambda=\frac{a}{m}(n+\sin \theta)$, where $a$ is the array periodicity, $m$ is an integer and $n$ the refractive index of air ${ }^{5}$. The absorption peaks at $\lambda \approx 620,780,1,030$ and $1,520 \mathrm{~nm}$ can be associated with the $m=5,4,3$ and 2 diffraction-coupled modes, respectively. The $m=4,5$ features each consist of two peaks because of a mismatch between the inverse polarizability and retarded dipole sum of individual nanoparticles in the plasmonic nanoarray in this spectral region ${ }^{29}$, caused by the presence of the hBN.

Figure $2 \mathrm{~b}$ plots $\Psi$ as a function of both wavelength and gate voltage $V_{\mathrm{g}}$, showing how the various features from the ultraviolet to near-infrared respond to electrical biasing of the device. As $V_{\mathrm{g}}$ is raised from 0 to $\pm 150 \mathrm{~V}$ we see dramatic changes in the FP resonances, along with a red-shift of the Rayleigh resonance wavelengths. These changes in reflection happen because of motion of the hBN/graphene heterostructure-the applied gate voltage creates an electrostatic force within the device, acting to reduce $d$. The Maxwell stresses caused by induced electrical charges can be of the order of 10 atmospheres (see Supplementary Note 1). We note that Casimir interactions between the graphene and gold are negligible at this length scale ${ }^{30}$. It is worth noting that, in general, the opto-electro-mechanical response of the structure is symmetric with respect to the sign of $V_{\mathrm{g}}$ and there is a threshold of $\pm 50 \mathrm{~V}$ before which application of $V_{\mathrm{g}}$ produces no change in optical reflection (no motion of the heterostructure). One exception to the above mechanism occurs near $\lambda=1.6 \mu \mathrm{m}$ for large negative $V_{\mathrm{g}}$. In this region the absorption changes because of electrical gating of graphene, moving its charge neutrality point and the spectral onset of optical Pauli blocking ${ }^{3}$. This results in an increase in the measured value of $\Psi$ at the highest wavelengths for the largest negative $V_{\mathrm{g}}$. It is worth noting that one can distinguish the effect of hBN motion and that of the Pauli blocking by the symmetry of the response (the effect of hBN motion is symmetric with respect to the sign of the applied voltage, whereas the Pauli blocking effect is not symmetric because of the initial doping of graphene) and by the wavelength range at which these effects are observed (see the discussion in Supplementary Note 2).

The response to $V_{\mathrm{g}}$ in the ultraviolet region measured in $s$-polarized reflection $\left(R_{\mathrm{s}}\right)$ is shown in Fig. 3a. Note that the hBN absorption feature at $\lambda=275 \mathrm{~nm}$ is relatively insensitive to $V_{\mathrm{g}}$ (and hence $d$ ) as the wavelength is approximately equal to the optical path length within the $\mathrm{hBN}$. However, as $V_{\mathrm{g}}$ is increased (and $d$ decreased) the adjacent FP absorption features are dramatically altered. This cavity modulation effect occurs over the entire measured ultraviolet range $(\lambda>250 \mathrm{~nm})$ and gives a peak modulation depth of $\sim 10 \%$ at $\lambda \sim 310 \mathrm{~nm}$. This compares favourably with prior attempts at ultraviolet modulation that have usually relied on wide band gaps ${ }^{31}$ or excitonic effects ${ }^{32}$ in semiconductor devices.

Moving to the visible and near-infrared ranges (for the same device) we have observed strong modulation of the diffractioncoupled resonances in the plasmonic nanoarray produced by graphene gating. Figure $3 \mathrm{~b}$ shows the changes in $p$-polarized reflection spectra for the $\lambda=780 \mathrm{~nm}$ resonance, whereas Fig. $3 c, d$ show $\Psi$ of $\sim 1,030$ and $1,520 \mathrm{~nm}$ respectively. In all three cases the reflection minima are shifted by up to $10 \mathrm{~nm}$ for $\left|V_{\mathrm{g}}\right|=150$ $\mathrm{V}-\mathrm{as}$ the $\mathrm{hBN}$ moves closer to the nanoarray the local fields increase and the refractive index of the ambient medium (ordinarily $n=1$ for air) is effectively changed. This in itself produces a strong modulation effect, simply because the plasmonic resonances are so narrow. Even though the minimum $\Psi$ value in the vicinity of $\lambda=1,520 \mathrm{~nm}$ (Fig. 3d) remains approximately constant $\left(\sim 26-27^{\circ}\right)$, its shifting wavelength with increasing $\left|V_{\mathrm{g}}\right|$ leads to a modulation depth of $20 \%$ at $\lambda=1,536 \mathrm{~nm}$. This is significantly higher than the near-infrared absolute modulation depths previously reported in grapheneand plasmonic-based devices $23,33-35$ : for example, Li et al. ${ }^{35}$ reported an absolute modulation depth of $\sim 9 \%$ at telecom wavelengths using a graphene-clad microfibre. The modulation frequency in our devices could be reasonably high $\sim 100 \mathrm{kHz}$ and could be further improved to $100 \mathrm{MHz}$ by a dedicated design (see the detailed discussions in Supplementary Notes 3 and 4).

Reflection spectra obtained experimentally are described well by rigorous coupled-wave analysis (RCWA) modelling (see Methods), in which the graphene/hBN heterostructure is displaced vertically, reducing $d$ from 300 to $200 \mathrm{~nm}$. The right-hand panels in Fig. 3a-d show the results of this modelling in the ultraviolet, visible and near-infrared regions of interest. Both the functional form and behaviour of the blue-shifting FP and red-shifting Rayleigh resonances are reproduced by the model. The differences between the experimental and modelled spectra can be attributed to impurities in, and roughness of, our gold nanostripe arrays. In addition, the height of the suspended area is not constant over the beam spot that leads to resonances smearing. Further modelling, of even smaller air gaps, reveals that as $d$ approaches zero the Rayleigh resonance wavelengths become increasingly sensitive to changes in $d$ (see Supplementary Fig. 3). With this knowledge future devices might be deliberately engineered with smaller initial air gaps, potentially greatly enhancing the achievable modulation depths.

Infrared reflection spectra were measured using Fourier transform infrared spectroscopy (see Methods). Figure 4a shows 

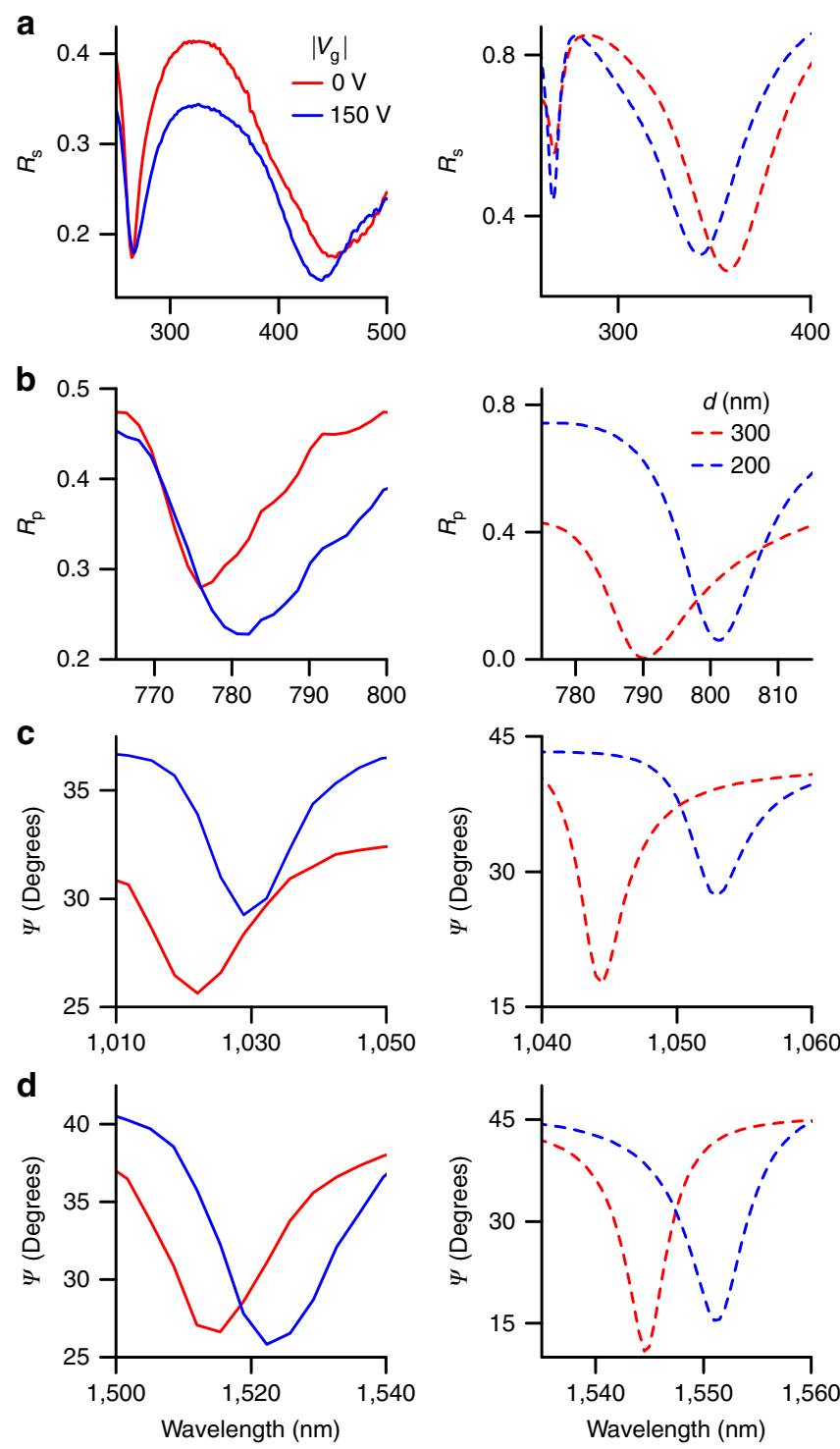

Figure 3 | Electromechanical modulation from ultraviolet to near-infra red regions. Measured reflection spectra (left column) of the graphene/ $\mathrm{hBN} /$ nanoarray for (a) $s$-polarized light $\left(R_{\mathrm{s}}\right)$ in the ultraviolet/blue range and (b) $p$-polarized light $\left(R_{\mathrm{p}}\right)$ in the visible. (c,d) Ellipsometric reflection in the near-infrared. All spectra were measured with $\theta=70^{\circ}$. Modelling results (right column) reveal that the electrically induced reflection modulation is explained by a changing air gap. The sharp reflection minima in (a) (centred at $\lambda=275 \mathrm{~nm}$ ) stems from the complex ultraviolet absorption spectrum of hBN. The features in (b-d) correspond to the fourth-, third- and second-order Rayleigh cutoff wavelengths of the nanoarray, respectively.

the strong modulation feature observed in the mid-infrared wavelength range produced by graphene gating. At such long wavelengths, incident light is not influenced by the grating structure, instead effectively experiencing a planar gold surface. On the long wavelength side of its Reststrahlen band, just beyond the transverse optic phonon wavelength of $\sim 7.35 \mu \mathrm{m}$, hBN displays strong light absorption and spectral dispersion. In this region we observe a broad reflection minimum, with reflection values falling to $33 \%$ at $\lambda \sim 7.6 \mu \mathrm{m}$ (Fig. $4 \mathrm{a}$ ). The electromechanical reduction of $d$ induces a narrowing of this feature and a blue shift of the reflection minimum by over $100 \mathrm{~nm}$. The reflection minimum also falls to $\sim 15 \%$. As a result, large absolute

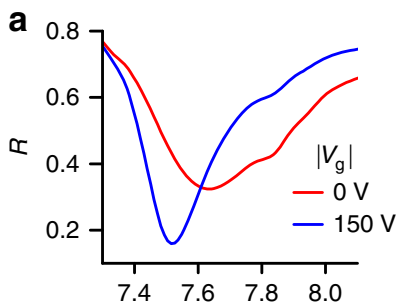

b
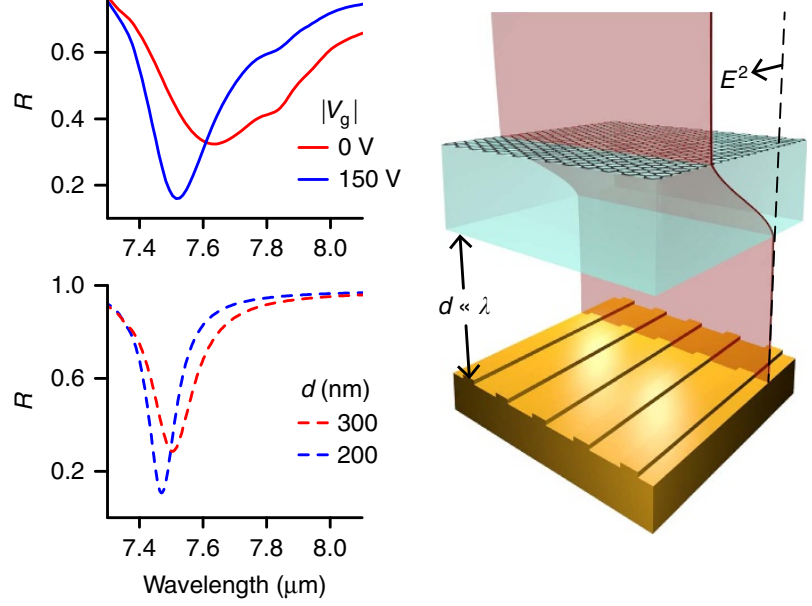

Figure 4 | Modulation and modelling of hexagonal boron nitride's Reststrahlen band. (a) Measured (upper panel) and modelled (lower panel) reflection spectra close to the transverse optical (TO) phonon energy in $\mathrm{hBN}$. The broader measured resonance width stems from the Fourier transform infrared (FTIR) reflecting objective that provides a range of incidence/measurement angles simultaneously $\left(\theta=12-24^{\circ}\right)$ compared with the modelled $\theta=15^{\circ}$. (b) The air gap is significantly smaller than the mid-IR wavelengths; however, close to the TO phonon energy, light is highly compressed within the hBN because of its large index of refraction.

reflection modulation depths are possible for a given wavelength - up to $30 \%$ at $\lambda=7.5 \mu \mathrm{m}$. This represents a dramatic improvement on existing mid-infrared graphene-plasmonic modulator results $22,36,37$ : for example, Yao et al. ${ }^{37}$ have previously reported an absolute reflection modulation depth of $\sim 20 \%$ at $\sim 7.6 \mu \mathrm{m}$. Comparing the device dimensions (total height $\sim 450-550 \mathrm{~nm}$ ) with the wavelength of this absorption feature reveals the high degree of light confinement within the structure. As a consequence of this confinement-on the order of $\sim \lambda / 10$ - the device is capable of modulating light with an optical interrogation volume of as little as $\lambda^{3} / 10$. (The actual modulation volume of our device was $\sim \lambda^{3}$ at mid-infrared frequencies. It can be reduced to the optical interrogation volume by using smaller suspended areas.)

As with shorter wavelengths, RCWA simulations agree very closely with the measured mid-infrared behaviour (Fig. 4a). The basic existence of the absorption feature at $\lambda \sim 7.5 \mu \mathrm{m}$ is attributable to material absorption in hBN. Hence, a similar reflection minimum also occurs in simulations of free-standing thin hBN films, and could be modulated simply by changing the hBN thickness (for example, from 110 to $140 \mathrm{~nm}$ ). However, the hBN thickness is fixed experimentally. Instead, we observe an analogous modulation effect arising from the motion of the hBN slab with respect to the gold mirror. Altering $d$ leads to changes in the optical field overlap with the hBN, as the field diminishes to zero at the gold surface (Fig. 4b). Although the nanostripe array was included in the above RCWA calculations, a much simpler Fresnel reflection-based model of a planar Au/air/hBN/graphene structure confirmed that it is not necessary for the observed midinfrared modulation.

\section{Discussion}

One potential application of this modulation technique is the study of the nanomechanics of suspended 2D materials. Our modulator design could be adapted to detect the motion of a $2 \mathrm{D}$ heterostructure by measuring the dispersive coupling strength $g=\Delta E / \Delta z$, where $\Delta E$ is the shift in the collective plasmon energy 
and $\Delta z$ the displacement of the membrane. In the case of a plasmonic nanoarray supporting diffraction-coupled plasmon resonances with the $2 \mathrm{D}$ heterostructure suspended close to its surface, this number was $g=4 \mathrm{meV} \mathrm{nm}^{-1}$ or $1,000 \mathrm{GHz} \mathrm{nm}^{-1}$ that is quite large for a plasmonic device.

In conclusion, we have shown that introducing an air gap of variable height into a graphene/hBN/nanoarray structure can allow for very strong optical reflection modulation via graphene gating. We have demonstrated that this effect can be used to create modulation from the mid-ultraviolet all the way to the upper Reststrahlen band of hBN (mid-infrared). The use of 2D atomic heterostructures allows for an extremely small optical interrogation volume of $\sim \lambda^{3} / 10$. To see such strong effects over such a broad spectral range in a device controlled by graphene gating is unprecedented and opens up many exciting possibilities in the development of optical nanoelectromechanical systems.

\section{Methods}

Nanoarray fabrication. Uniform arrays of gold nanostripes (total area $=300$ $\times 100 \mu \mathrm{m}$ ) were fabricated (with a gold sublayer) on glass substrates using standard electron beam lithography and electron beam evaporation techniques ${ }^{9,26}$. First, the glass substrate was covered with a thin film of $\mathrm{Cr}(3 \mathrm{~nm})$ to prevent charging during lithography, followed a flat region of $\mathrm{Cr}$ ( $3 \mathrm{~nm}$, for adhesion) and $\mathrm{Au}(65 \mathrm{~nm})$. As well as forming the metallic sublayer, this region acted as the back contact for electrical measurements. Next, the array of gold nanostripes (length $100 \mu \mathrm{m})$ was fabricated on top of the gold sublayer, once again using a $\mathrm{Cr}(3 \mathrm{~nm})$ adhesion layer. Finally, the exposed areas of the initial $\mathrm{Cr}$ film were removed via wet chemical etching. Nanostripe dimensions were confirmed using scanning electron microscopy.

Graphene and boron nitride transfer. Single-layered graphene and hBN were prepared by standard micromechanical exfoliation ${ }^{1}$. hBN was obtained from the Advanced Materials Laboratory, National Institute for Materials Science, Japan. The $\mathrm{hBN}$ and graphene flakes (both $\sim 50 \times 100 \mu \mathrm{m}$ ) were deposited onto separate oxidized Si wafers. Each flake was then delaminated by spinning a layer of poly(methyl methacrylate) on top of the substrate and etching away the oxide layer. Sequential flake transfers to the nanoarray were performed using optical microscopemounted micromanipulator. After each transfer the poly(methyl methacrylate) membrane was dissolved with acetone. Finally, the graphene/hBN/gold nanoarray was annealed in an $\mathrm{Ar} / \mathrm{H}_{2}$ gas mixture at $275^{\circ} \mathrm{C}$ for $3 \mathrm{~h}$. Adhesion between the $\mathrm{hBN}$ and rough nanoarray surface was non-uniform over the flake area, leading to the existence of the required air gap in regions of each device. The nanostripe array period is significantly larger than width of the individual nanostripes, resulting in a small contact area between the hBN and nanoarray surface. The gaps in physical contact have a natural tendency to propagate, leading to a high probability of the formation of air gaps (bubbles) during heterostructure transfer.

Optical and electrical measurements. Ellipsometric and reflection spectra were measured in a Woollam M-2000F focussed beam ellipsometer (Numerical Aperture 0.1$)$. At the highest angles of incidence $\left(\sim 80^{\circ}\right)$, with the strongest localized plasmon resonances), the measurement spot size was larger than the graphene/hBN area; the angle of incidence was reduced to $70^{\circ}$ to ensure the measurement spot was incident only on the active device area. Ellipsometry characterizes a sample's optical properties via the ellipsometric parameters $\Psi$ (ellipsometric reflection) and $\Delta$ (ellipsometric phase shift) that are related by the equation $\tan (\Psi) \exp (i \Delta)=r_{\mathrm{p}} / r_{\mathrm{s}}$, where $r_{\mathrm{p}}$ and $r_{\mathrm{s}}$ are the amplitude reflection coefficients for $p$ - and $s$-polarized light, respectively. Ellipsometry measures the ratio of reflection coefficients, making it immune to many sources of noise and therefore a highly sensitive technique. Fourier transform infrared spectroscopy was performed using a Bruker Vertex 80 system with a Hyperion 3000 microscope. A variety of sources and detectors combined with aluminium-coated reflective optics enable this system to be used from visible to $\mathrm{THz}$ wavelengths. A reflecting objective provides a range of incident angles $\left(\theta=12-24^{\circ}\right)$ and the entire beam path purged with dry, $\mathrm{CO}_{2}$-scrubbed air to minimize strong atmospheric infrared absorption bands. Gate voltages were applied using a Keithley 2400 Sourcemeter. All measurements were performed under standard room temperature and pressure conditions.

Modelling. Simulations were performed using the Reticolo package in Matlab ${ }^{38}$ that relies upon a RCWA method ${ }^{39}$. The optical properties of Au were calculated within this package using the Lorentz-Drude model and the dispersion relation of $\mathrm{hBN}$ was calculated using the Lorentz oscillator model ${ }^{40}$

$$
\varepsilon(\omega)=\varepsilon_{\infty}\left(1+\frac{\omega_{\mathrm{LO}}^{2}-\omega_{\mathrm{TO}}^{2}}{\omega_{\mathrm{TO}}^{2}-\omega^{2}-i \gamma \omega}\right)
$$

where $\omega_{\mathrm{LO}}$ and $\omega_{\mathrm{TO}}$ are the longitudinal optical and transverse optical phonon frequencies respectively, $\varepsilon_{\infty}$ is the high frequency permittivity and $\gamma$ is the damping constant associated with the plasma oscillation. An additional spectral absorption feature was introduced to the modelled $\mathrm{hBN}$ in order to reproduce the experimentally observed absorption peak in the near-ultraviolet region. Graphene optical conductivity was taken in random-phase approximation ${ }^{12}$.

Data availability. The data that support the findings of this study are available from the corresponding author upon request.

\section{References}

1. Novoselov, K. S. et al. Two-dimensional atomic crystals. Proc. Natl Acad. Sci. USA 102, 10451-10453 (2005).

2. Bonaccorso, F., Sun, Z., Hasan, T. \& Ferrari, A. C. Graphene photonics and optoelectronics. Nat. Photon. 4, 611-622 (2010).

3. Grigorenko, A. N., Polini, M. \& Novoselov, K. S. Graphene plasmonics. Nat. Photon. 6, 749-758 (2012).

4. Barnes, W. L., Dereux, A. \& Ebbesen, T. W. Surface plasmon subwavelength optics. Nature 424, 824-830 (2003).

5. Kravets, V. G., Schedin, F. \& Grigorenko, A. N. Extremely narrow plasmon resonances based on diffraction coupling of localized plasmons in arrays of metallic nanoparticles. Phys. Rev. Lett. 101, 087403 (2008).

6. Auguié, B. \& Barnes, W. L. Collective resonances in gold nanoparticle arrays. Phys. Rev. Lett. 101, 143902 (2008).

7. Zou, S., Janel, N. \& Schatz, G. C. Silver nanoparticle array structures that produce remarkably narrow plasmon lineshapes. J. Chem. Phys. 120, 10871-10875 (2004).

8. Gramotnev, D. K. \& Bozhevolnyi, S. I. Plasmonics beyond the diffraction limit. Nat. Photon. 4, 83-91 (2010).

9. Kravets, V. G. et al. Singular phase nano-optics in plasmonic metamaterials for label-free single-molecule detection. Nat. Mater. 12, 304-309 (2013).

10. García de Abajo, F. J. Graphene nanophotonics. Science 339, 917-918 (2013).

11. Nair, R. R. et al. Fine structure constant defines visual transparency of graphene. Science 320, 1308-1308 (2008).

12. Koppens, F. H. L., Chang, D. E. \& García de Abajo, F. J. Graphene plasmonics: a platform for strong light-matter interactions. Nano Lett. 11, 3370-3377 (2011).

13. Chen, J. et al. Optical nano-imaging of gate-tunable graphene plasmons. Nature 487, 77-81 (2012).

14. Geim, A. K. \& Grigorieva, I. V. Van der Waals heterostructures. Nature 499, 419-425 (2013).

15. Withers, F. et al. Light-emitting diodes by band-structure engineering in van der Waals heterostructures. Nat. Mater. 14, 301-306 (2015).

16. Britnell, L. et al. Strong light-matter interactions in heterostructures of atomically thin films. Science 340, 1311-1314 (2013).

17. Echtermeyer, T. J. et al. Strong plasmonic enhancement of photovoltage in graphene. Nat. Commun. 2, 458 (2011).

18. Schedin, F. et al. Surface-enhanced Raman spectroscopy of graphene. ACS Nano 4, 5617-5626 (2010).

19. Wang, F. et al. Gate-variable optical transitions in graphene. Science 320, 206-209 (2008).

20. Ju, L. et al. Graphene plasmonics for tunable terahertz metamaterials. Nat. Nanotechnol 6, 630-634 (2011).

21. Kim, J. et al. Electrical control of optical plasmon resonance with graphene. Nano Lett. 12, 5598-5602 (2012).

22. Yao, Y. et al. Wide wavelength tuning of optical antennas on graphene with nanosecond response time. Nano Lett. 14, 214-219 (2014)

23. Ansell, D. et al. Hybrid graphene plasmonic waveguide modulators. Nat. Commun. 6, 8846 (2015).

24. Dennis, B. S. et al. Compact nanomechanical plasmonic phase modulators. Nat. Photon. 9, 267-273 (2015).

25. Reserbat-Plantey, A. et al. Electromechanical control of nitrogen-vacancy defect emission using graphene NEMS. Nat. Commun. 7, 10218 (2016).

26. Thackray, B. D. et al. Super-narrow, extremely high quality collective plasmon resonances at telecom wavelengths and their application in a hybrid graphene-plasmonic modulator. Nano Lett. 15, 3519-3523 (2015).

27. Dean, C. R. et al. Boron nitride substrates for high-quality graphene electronics Nat. Nanotechnol. 5, 722-726 (2010).

28. Xu, Y.-N. \& Ching, W. Y. Calculation of ground-state and optical properties of boron nitrides in the hexagonal, cubic, and wurtzite structures. Phys. Rev. B $\mathbf{4 4}$ 7787-7798 (1991).

29. Thackray, B. D. et al. Narrow collective plasmon resonances in nanostructure arrays observed at normal light incidence for simplified sensing in asymmetric air and water environments. ACS Photonics 1, 1116-1126 (2014).

30. Bordag, M., Fialkovsky, I., Gitman, D. \& Vassilevich, D. Casimir interaction between a perfect conductor and graphene described by the Dirac model. Phys Rev. B 80, 245406 (2009). 
31. Wraback, M., Shen, H., Liang, S., Gorla, C. R. \& Lu, Y. High contrast, ultrafast optically addressed ultraviolet light modulator based upon optical anisotropy in ZnO films grown on R-plane sapphire. Appl. Phys. Lett. 74, 507-509 (1999).

32. Oberhofer, A. E. et al. Planar gallium nitride ultraviolet optical modulator. Appl. Phys. Lett. 83, 2748-2750 (2003).

33. Thijssen, R., Verhagen, E., Kippenberg, T. J. \& Polman, A. Plasmon nanomechanical coupling for nanoscale transduction. Nano Lett. 13, 3293-3297 (2013).

34. Youngblood, N., Anugrah, Y., Ma, R., Koester, S. J. \& Li, M. Multifunctional graphene optical modulator and photodetector integrated on silicon waveguides. Nano Lett. 14, 2741-2746 (2014).

35. Li, W. et al. Ultrafast all-optical graphene modulator. Nano Lett. 14, 955-959 (2014).

36. Dabidian, N. et al. Electrical switching of infrared light using graphene integration with plasmonic Fano resonant metasurfaces. ACS Photonics 2, 216-227 (2015).

37. Yao, Y. et al. Broad electrical tuning of graphene-loaded plasmonic antennas. Nano Lett. 13, 1257-1264 (2013).

38. Hugonin, J. P. \& Lalanne, P. Reticolo Software for Grating Analysis (Institut d'Optique, 2005).

39. Moharam, M. G. \& Gaylord, T. K. Rigorous coupled-wave analysis of metallic surface-relief gratings. J. Opt. Soc. Am. A 3, 1780-1787 (1986).

40. Caldwell, J. D. et al. Low-loss, infrared and terahertz nanophotonics using surface phonon polaritons. Nanophotonics 4, 2192-8614 (2015).

\section{Acknowledgements}

This work has been supported by the European Commission under the Graphene Flagship (Contract No. CNECT-ICT-604391), Bluestone Global Tech and EPSRC Grant EP/K011022/1. P.A.T. and G.H.A. acknowledge support from the North-West

Nanoscience Doctoral Training Centre, EPSRC Grant EP/G03737X/1.

\section{Author contributions}

A.N.G. conceived the project. F.J.R., G.H.A., P.A.T. and V.G.K. made the devices. P.A.T., O.P.M., D.K., Y.S., F.J.R. and V.G.K. performed measurements. O.P.M. did the modelling. All authors contributed to the discussion of the project and writing of the manuscript.

\section{Additional information}

Supplementary Information accompanies this paper at http://www.nature.com/ naturecommunications

Competing financial interests: The authors declare no competing financial interests.

Reprints and permission information is available online at http://npg.nature.com/ reprintsandpermissions/

How to cite this article: Thomas, P. A. et al. Nanomechanical electro-optical modulator based on atomic heterostructures. Nat. Commun. 7:13590 doi: 10.1038/ncomms13590 (2016).

Publisher's note: Springer Nature remains neutral with regard to jurisdictional claims in published maps and institutional affiliations.

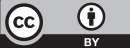

This work is licensed under a Creative Commons Attribution 4.0 International License. The images or other third party material in this article are included in the article's Creative Commons license, unless indicated otherwise in the credit line; if the material is not included under the Creative Commons license, users will need to obtain permission from the license holder to reproduce the material To view a copy of this license, visit http://creativecommons.org/licenses/by/4.0/

(C) The Author(s) 2016 